\title{
Boundary Value Problems for the Quaternionic Hermitian System on Fractal Hypersurfaces in $\mathbb{R}^{4 n}$
}

\author{
Ricardo Abreu-Blaya Juan Bory-Reyes Fred Brackx \\ Hennie De Schepper Tania Moreno-García \\ Frank Sommen
}

\begin{abstract}
In this paper the Dirichlet and jump problems on fractal hypersurfaces in $\mathbb{R}^{4 n}$ for quaternionic Hermitian monogenic functions are presented using a circulant matrix approach. We introduce the matrix Cauchy and Hilbert transforms on fractal boundaries of bounded domains and prove a matrix Cauchy formula.
\end{abstract}

Keywords. Hermitian Clifford analysis, fractals, boundary value problems.

Mathematics Subject Classification (2000). 30G35.

\section{Introduction}

Clifford analysis [7] offers an efficient framework to study higher dimensional boundary value problems in Euclidean space. Recently the notions of complex and of quaternionic Hermitian Clifford analysis were introduced, refining the classical orthogonal framework. The complex and quaternionic Hermitian monogenic functions are in the kernel of two complex, respectively four quaternionic, Dirac operators. The theory of complex Hermitian monogenicity is becoming well-established, while the quaternionic hermitian monogenicty is still under full development, we refer e.g. to $[8,9,10,11,23]$ for the complex setting and $[13,14,15,22]$ for the quaternionic one.

In [2], Borel-Pompeiu and Cauchy integral formulas on Lyapunov surfaces in the quaternionic Hermitian setting by means of a $(4 \times 4)$ circulant matrix approach were established, which can be thought as similar in spirit to the circulant $(2 \times 2)$ matrix approach introduced in [12] in complex Hermitian Clifford analysis. Subsequently in [3] a quaternionic Hermitian Cauchy integral was introduced and its 
boundary values were studied, leading to a matrix quaternionic Hermitian Hilbert transform. These quaternionic Cauchy and Hilbert transforms provide a useful tool for studying boundary value problems for the quaternionic Hermitian system. In [4] a Cauchy transform on fractal surfaces was introduced in the complex Hermitian case. A Hilbert transform on fractal surfaces in the quaternionic Hermitian case is presented in [5].

In this paper we deal with fractal boundaries and the main problem that we address is the problem of finding a quaternionic Hermitian monogenic function with a given jump on a given fractal surface of $\mathbb{R}^{4 n}$. Additionally, Dirichlet type problems for the quaternionic Hermitian system are considered.

\section{Preliminaries}

Let $\left(e_{1}, \ldots, e_{m}\right)$ be an orthonormal basis of Euclidean space $\mathbb{R}^{m}$ and consider the real Clifford algebra $\mathbb{R}_{0, m}$ constructed over $\mathbb{R}^{m}$. The non-commutative multiplication in $\mathbb{R}_{0, m}$ is governed by the rules:

$$
\begin{aligned}
e_{j}^{2} & =-1, & & j=1, \ldots, m \\
e_{j} e_{k}+e_{k} e_{j} & =0, & & j \neq k
\end{aligned}
$$

In $\mathbb{R}_{0, m}$ one can consider the following automorphisms:

(i) the conjugation for which $\bar{e}_{r}=-e_{r}$ and $\overline{a b}=\bar{b} \bar{a}$ for all $a, b \in \mathbb{R}_{m}$

(ii) the main involution for which $\tilde{e}_{r}=-e_{r}$ and $\tilde{a b}=\tilde{a} \tilde{b}$ for all $a, b \in \mathbb{R}_{m}$

Let us consider the skew-field of quaternions $\mathbb{H}$ whose elements will be denoted by $q=x_{0}+i x_{1}+j x_{2}+k x_{3}$ with $i^{2}=j^{2}=k^{2}=-1$ and $i j=-j i=k$. The algebra of real quaternions may be identified with the Clifford algebra $\mathbb{R}_{0,2}$ through $i \leftrightarrow e_{1}$, $j \leftrightarrow e_{2}$ and $k \leftrightarrow e_{1} e_{2}$. This isomorphism leads to the conjugation

$$
\bar{q}=x_{0}-i x_{1}-j x_{2}-k x_{3} .
$$

and to the main involution

$$
q^{\gamma}:=\tilde{q}=x_{0}-i x_{1}-j x_{2}+k x_{3} .
$$

However, it is natural to introduce two more versions of the main involution defined by

$$
\begin{aligned}
q^{\alpha} & =x_{0}+i x_{1}-j x_{2}-k x_{3}, \\
q^{\beta} & =x_{0}-i x_{1}+j x_{2}-k x_{3} .
\end{aligned}
$$

Definition 1 ([22]) If the dimension $m$ is a multiple of 4 , say $m=4 n$, the quaternionic Witt basis of $\mathbb{H}_{4 n}=\mathbb{H} \otimes_{\mathbb{R}} \mathbb{R}_{0,4 n}$ is given by $\left\{f_{l}, f_{l}^{\alpha}, f_{l}^{\beta}, f_{l}^{\gamma}\right\}, l=$ $1, \ldots, n$, where

$$
f_{l}=e_{1+4(l-1)}-i e_{2+4(l-1)}-j e_{3+4(l-1)}-k e_{4+4(l-1)},
$$




$$
\begin{aligned}
& f_{l}^{\alpha}=e_{1+4(l-1)}-i e_{2+4(l-1)}+j e_{3+4(l-1)}+k e_{4+4(l-1)}, \\
& f_{l}^{\beta}=e_{1+4(l-1)}+i e_{2+4(l-1)}-j e_{3+4(l-1)}+k e_{4+4(l-1)}, \\
& f_{l}^{\gamma}=e_{1+4(l-1)}+i e_{2+4(l-1)}+j e_{3+4(l-1)}-k e_{4+4(l-1)} .
\end{aligned}
$$

Conversely it is possible to express the initial orthogonal basis $\left(e_{r}, r=1, \ldots, 4 n\right)$ in terms of the quaternionic Witt basis vectors $\left(f_{l}, f_{l}^{\alpha}, f_{l}^{\beta}, f_{l}^{\gamma}, l=1, \ldots, n\right)$.

\subsection{The quaternionic Hermitian vector derivative}

Let us define the following Clifford vectors

$$
\begin{gathered}
\underline{X}=\underline{X}_{0}=\sum_{l=1}^{n}\left(e_{4 l-3} x_{4 l-3}+e_{4 l-2} x_{4 l-2}+e_{4 l-1} x_{4 l-1}+e_{4 l} x_{4 l}\right) \\
\underline{X}_{1}=\sum_{l=1}^{n}\left(e_{4 l-3} x_{4 l-2}-e_{4 l-2} x_{4 l-3}-e_{4 l-1} x_{4 l}+e_{4 l} x_{4 l-1}\right) \\
\underline{X}_{2}=\sum_{l=1}^{n}\left(e_{4 l-3} x_{4 l-1}+e_{4 l-2} x_{4 l}-e_{4 l-1} x_{4 l-3}-e_{4 l} x_{4 l-2}\right) \\
\underline{X}_{3}=\sum_{l=1}^{n}\left(e_{4 l-3} x_{4 l}-e_{4 l-2} x_{4 l-1}+e_{4 l-1} x_{4 l-2}-e_{4 l} x_{4 l-3}\right)
\end{gathered}
$$

A straightforward computation shows that

$$
\begin{aligned}
\underline{X}_{r}^{2} & =-\left|\underline{X}_{0}\right|^{2}, & & r=0, \ldots, 3 \\
\underline{X}_{r} \underline{X}_{s}+\underline{X}_{s} \underline{X}_{r} & =0, & & r \neq s
\end{aligned}
$$

We can introduce four Dirac type operators as Fischer duals of the vectors $\underline{X}_{0}, \underline{X}_{1}, \underline{X}_{2}, \underline{X}_{3}$; they are then given by

$$
\begin{aligned}
& \partial_{\underline{X}_{0}}=\sum_{l=1}^{n}\left(e_{4 l-3} \partial_{x_{4 l-3}}+e_{4 l-2} \partial_{x_{4 l-2}}+e_{4 l-1} \partial_{x_{4 l-1}}+e_{4 l} \partial_{x_{4 l}}\right) \\
& \partial_{\underline{X}_{1}}=\sum_{l=1}^{n}\left(e_{4 l-3} \partial_{x_{4 l-2}}-e_{4 l-2} \partial_{x_{4 l-3}}-e_{4 l-1} \partial_{x_{4 l}}+e_{4 l} \partial_{x_{4 l-1}}\right) \\
& \underline{\underline{X}}_{2}=\sum_{l=1}^{n}\left(e_{4 l-3} \partial_{x_{4 l-1}}+e_{4 l-2} \partial_{x_{4 l}}-e_{4 l-1} \partial_{x_{4 l-3}}-e_{4 l} \partial_{x_{4 l-2}}\right) \\
& \partial_{\underline{X}_{3}}=\sum_{l=1}^{n}\left(e_{4 l-3} \partial_{x_{4 l}}-e_{4 l-2} \partial_{x_{4 l-1}}+e_{4 l-1} \partial_{x_{4 l-2}}-e_{4 l} \partial_{x_{4 l-3}}\right)
\end{aligned}
$$


By Fischer duality we obtain from (1) and (2) the relations

$$
\begin{aligned}
\partial_{\underline{X}_{r}} \partial_{\underline{X}_{r}} & =-\Delta_{4 n}, & & r=0, \ldots, 3 \\
\partial_{\underline{X}_{r}} \partial_{\underline{X}_{s}}+\partial_{\underline{X}_{s}} \partial_{\underline{X}_{r}} & =0, & & r \neq s
\end{aligned}
$$

Next we define the quaternionic Hermitian variables

$$
\begin{gathered}
\underline{Z}=\underline{Z}_{0}=\underline{X}_{0}+i \underline{X}_{1}+j \underline{X}_{2}+k \underline{X}_{3} \\
\underline{Z}_{1}=\underline{X}_{0}+i \underline{X}_{1}-j \underline{X}_{2}-k \underline{X}_{3} \\
\underline{Z}_{2}=\underline{X}_{0}-i \underline{X}_{1}+j \underline{X}_{2}-k \underline{X}_{3} \\
\underline{Z}_{3}=\underline{X}_{0}-i \underline{X}_{1}-j \underline{X}_{2}+k \underline{X}_{3}
\end{gathered}
$$

For further use, observe that

$$
\underline{Z}_{0} \underline{Z}_{0}+\underline{Z}_{1} \underline{Z}_{1}+\underline{Z}_{2} \underline{Z}_{2}+\underline{Z}_{3} \underline{Z}_{3}=-8\left|\underline{X}_{0}\right|^{2}
$$

and

$$
\underline{Z}_{0} \underline{Z}_{0}^{\dagger}+\underline{Z}_{1} \underline{Z}_{1}^{\dagger}+\underline{Z}_{2} \underline{Z}_{2}^{\dagger}+\underline{Z}_{3} \underline{Z}_{3}^{\dagger}=16\left|\underline{X}_{0}\right|^{2},
$$

where $(.)^{\dagger}$ denotes the Hermitian quaternionic conjugation, i.e. the composition of the conjugation in the Clifford algebra $\mathbb{R}_{4 n}$ and conjugation in the quaternions $\mathbb{H}$

The Hermitian Dirac operators are similarly derived from the orthogonal Dirac operators:

$$
\begin{aligned}
& \partial_{\underline{Z}_{0}}=\frac{1}{16}\left(\partial_{\underline{X}_{0}}+i \partial_{\underline{X}_{1}}+j \partial_{\underline{X}_{2}}+k \partial_{\underline{X}_{3}}\right) \\
& \partial_{\underline{Z}_{1}}=\frac{1}{16}\left(\partial_{\underline{X}_{0}}+i \partial_{\underline{X}_{1}}-j \partial_{\underline{X}_{2}}-k \partial_{\underline{X}_{3}}\right) \\
& \partial_{\underline{Z}_{2}}=\frac{1}{16}\left(\partial_{\underline{X}_{0}}-i \partial_{\underline{X}_{1}}+j \partial_{\underline{X}_{2}}-k \partial_{\underline{X}_{3}}\right) \\
& \partial_{\underline{Z}_{3}}=\frac{1}{16}\left(\partial_{\underline{X}_{0}}-i \partial_{\underline{X}_{1}}-j \partial_{\underline{X}_{2}}+k \partial_{\underline{X}_{3}}\right)
\end{aligned}
$$

The Laplacian in $\mathbb{R}^{4 n}$ is strongly related to the quaternionic Hermitian Dirac operators; for example there holds:

$$
\Delta_{4 n}=16\left(\partial_{\underline{Z}_{0}} \partial_{\underline{Z}_{0}}^{\dagger}+\partial_{\underline{Z}_{1}} \partial_{\underline{Z}_{1}}^{\dagger}+\partial_{\underline{Z}_{2}} \partial_{\underline{Z}_{2}}^{\dagger}+\partial_{\underline{Z}_{3}} \partial_{\underline{Z}_{3}}^{\dagger}\right)
$$

\subsection{Hermitian monogenic functions in $\mathbb{R}^{4 n}$}

The following definition stems from [22]. 
Definition 2 Let $\Omega$ be an open set in $\mathbb{R}^{m}, m=4 n$. A continuously differentiable function $f: \Omega \mapsto \mathbb{H}_{m}$ is said to be (left) q-Hermitian monogenic in $\Omega$ if it satisfies the system of equations

$$
\partial_{\underline{Z}_{0}} f=\partial_{\underline{Z}_{1}} f=\partial_{\underline{Z}_{2}} f=\partial_{\underline{Z}_{3}} f=0
$$

or, equivalently, the system

$$
\partial_{\underline{X}_{0}} f=\partial_{\underline{X}_{1}} f=\partial_{\underline{X}_{2}} f=\partial_{\underline{X}_{3}} f=0
$$

Note that an alternative definition of $q$-Hermitian monogenic functions is given in [15], which is proved in [13] to be equivalent with the concept mentioned above.

In a similar way right $q$-Hermitian monogenicity is defined. Functions which are both left and right $q$-Hermitian monogenic are called two-sided $q$-Hermitian monogenic.

The fundamental solutions of the Dirac operators $\partial_{\underline{X}_{0}}, \partial_{\underline{X}_{1}}, \partial_{\underline{X}_{2}}, \partial_{\underline{X}_{3}}$, i.e. the orthogonal Cauchy kernels, are respectively given by

$$
\begin{aligned}
& E_{0}(\underline{X})=-\frac{1}{a_{4 n}} \frac{\underline{X}_{0}}{|\underline{X}|^{4 n}} \\
& E_{1}(\underline{X})=-\frac{1}{a_{4 n}} \frac{\underline{X}_{1}}{|\underline{X}|^{4 n}} \\
& E_{2}(\underline{X})=-\frac{1}{a_{4 n}} \frac{\underline{X}_{2}}{|\underline{X}|^{4 n}} \\
& E_{3}(\underline{X})=-\frac{1}{a_{4 n}} \frac{\underline{X}_{3}}{|\underline{X}|^{4 n}}
\end{aligned}
$$

where $a_{4 n}$ denotes the area of the unit sphere $S^{4 n-1}$ in $\mathbb{R}^{4 n}$.

Explicitly, this means that in distributional sense

$$
\partial_{\underline{X}_{0}} E_{0}(\underline{X})=\partial_{\underline{X}_{1}} E_{1}(\underline{X})=\partial_{\underline{X}_{2}} E_{2}(\underline{X})=\partial_{\underline{X}_{3}} E_{3}(\underline{X})=\delta(\underline{X}) .
$$

Similarly as above, we now introduce the Hermitian Cauchy kernels given by

$$
\begin{aligned}
& \mathcal{E}_{0}=E_{0}-i E_{1}-j E_{2}-k E_{3} \\
& \mathcal{E}_{1}=E_{0}-i E_{1}+j E_{2}+k E_{3} \\
& \mathcal{E}_{2}=E_{0}+i E_{1}-j E_{2}+k E_{3} \\
& \mathcal{E}_{3}=E_{0}+i E_{1}+j E_{2}-k E_{3}
\end{aligned}
$$

or, more explicitly

$$
\mathcal{E}_{0}(\underline{Z})=\frac{1}{a_{4 n}} \frac{\underline{Z}_{0}^{\dagger}}{|\underline{Z}|^{4 n}}
$$




$$
\begin{aligned}
& \mathcal{E}_{1}(\underline{Z})=\frac{1}{a_{4 n}} \frac{\underline{Z}_{1}^{\dagger}}{|\underline{Z}|^{4 n}} \\
& \mathcal{E}_{2}(\underline{Z})=\frac{1}{a_{4 n}} \frac{\underline{Z}}{|\underline{Z}|^{4 n}} \\
& \mathcal{E}_{3}(\underline{Z})=\frac{1}{a_{4 n}} \frac{\underline{Z}}{|\underline{Z}|^{4 n}}
\end{aligned}
$$

Note that $\mathcal{E}_{0}, \mathcal{E}_{1}, \mathcal{E}_{2}$ and $\mathcal{E}_{3}$ are not the fundamental solutions of the respective Hermitian Dirac operators $\partial_{\underline{Z}_{0}}, \partial_{\underline{Z}_{1}}, \partial_{\underline{Z}_{2}}$ and $\partial_{\underline{Z}_{3}}$. However, the following theorem, proved in [2], justifies the consideration of such kernels.

Theorem 1 Introducing the circulant $(4 \times 4)$ matrices

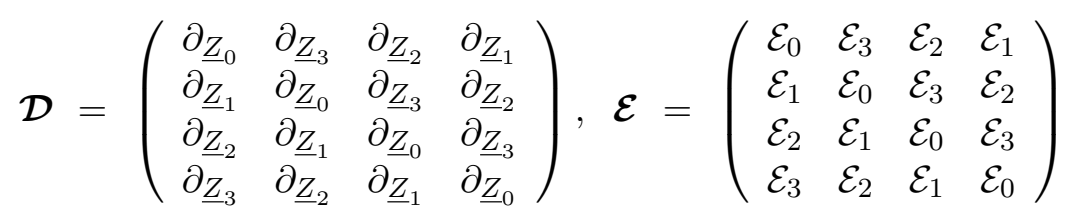

and

$$
\boldsymbol{\delta}=\left(\begin{array}{llll}
\delta & 0 & 0 & 0 \\
0 & \delta & 0 & 0 \\
0 & 0 & \delta & 0 \\
0 & 0 & 0 & \delta
\end{array}\right)
$$

one obtains that

$$
\mathcal{D}^{T} \mathcal{E}=\mathcal{E D}^{T}=\boldsymbol{\delta}
$$

Here and subsequently we denote the transpose of a circulant matrix $\mathbf{A}$ by $\mathbf{A}^{T}$.

Therefore, $\mathcal{E}$ may be considered as a fundamental solution of $\mathcal{D}$, when this concept is reinterpreted in a matricial context.

We associate, with functions $g_{0}, g_{1}, g_{2}$ and $g_{3}$ defined in $\Omega \subset \mathbb{R}^{4 n}$ and taking values in $\mathbb{H}_{4 n}$, the $(4 \times 4)$ circulant matrix function

$$
\boldsymbol{G}=\left(\begin{array}{cccc}
g_{0} & g_{3} & g_{2} & g_{1} \\
g_{1} & g_{0} & g_{3} & g_{2} \\
g_{2} & g_{1} & g_{0} & g_{3} \\
g_{3} & g_{2} & g_{1} & g_{0}
\end{array}\right)
$$

We recall that any circulant matrix $\mathbf{A}$ is fully specified by one vector, which appears as the first column of $\mathbf{A}$. Thus, to avoid repetitions and for the sake of brevity we use frequently in this paper the notation

$$
\boldsymbol{G}=\operatorname{circ}\left(\begin{array}{c}
g_{0} \\
g_{1} \\
g_{2} \\
g_{3}
\end{array}\right)
$$


To avoid confusion, we shall use small letters to denote $\mathbb{H}_{4 n}$-valued functions and boldface capital letters to denote $(4 \times 4)$ circulant matrix functions. We say that $\boldsymbol{G}$ belongs to some standard class of function if each of its entries belongs to that class.

In this manner, the spaces of $k$-time continuous differentiable, $\alpha$-Hölder continuous $(0<\alpha \leq 1)$ and $p$-integrable functions $((4 \times 4)$ circulant matrix functions $)$ are denoted by $C^{k}(\mathbf{E})\left(\mathbf{C}^{k}(\mathbf{E})\right), C^{0, \alpha}(\mathbf{E})\left(\mathbf{C}^{0, \alpha}(\mathbf{E})\right)$ and $L^{p}(\mathbf{E})\left(\mathbf{L}^{p}(\mathbf{E})\right)$ respectively, where $\mathbf{E}$ can be any suitable subset of $\mathbb{R}^{4 n}$.

However, introducing the non-negative function

$$
\|\boldsymbol{G}(\underline{X})\|=\max \left\{\left|g_{0}(\underline{X})\right|,\left|g_{1}(\underline{X})\right|,\left|g_{2}(\underline{X})\right|,\left|g_{3}(\underline{X})\right|\right\}
$$

the classes $\mathbf{C}^{0, \alpha}(\mathbf{E})$ and $\mathbf{L}^{p}(\mathbf{E})$ may also be defined by means of the traditional estimates

$$
\|\boldsymbol{G}\|_{\alpha}=\sup _{\underline{X}, \underline{Y} \in \mathbf{E}} \frac{\|\boldsymbol{G}(\underline{X})-\boldsymbol{G}(\underline{Y})\|}{|\underline{X}-\underline{Y}|^{\alpha}}<+\infty
$$

and

$$
\|\boldsymbol{G}\|_{p}=\left(\int_{\mathbf{E}}\|\boldsymbol{G}(\underline{X})\|^{p}\right)^{\frac{1}{p}}<+\infty
$$

respectively.

Definition 3 Let $\Omega$ be an open set in $\mathbb{R}^{4 n}$. We call $\boldsymbol{G}$ left (respectively right) QHermitian monogenic in $\Omega$ if and only if it satisfies in $\Omega \mathcal{D}^{T} \boldsymbol{G}=\boldsymbol{O}$ (respectively $\boldsymbol{G D}^{T}=\boldsymbol{O}$ ). Here $\boldsymbol{O}$ denotes the matrix with zero entries. Matrix functions which are both left and right $\mathbf{Q}$-Hermitian monogenic are called two-sided $\mathbf{Q}$-Hermitian monogenic.

The system $\mathcal{D}^{T} \boldsymbol{G}=\boldsymbol{O}$ explicitly reads

$$
\begin{aligned}
& \partial_{\underline{Z}_{0}} g_{0}+\partial_{\underline{Z}_{1}} g_{1}+\partial_{\underline{Z}_{2}} g_{2}+\partial_{\underline{Z}_{3}} g_{3}=0 \\
& \partial_{\underline{Z}_{3}} g_{0}+\partial_{\underline{Z}_{0}} g_{1}+\partial_{\underline{Z}_{1}} g_{2}+\partial_{\underline{Z}_{2}} g_{3}=0 \\
& \partial_{\underline{Z}_{2}} g_{0}+\partial_{\underline{Z}_{3}} g_{1}+\partial_{\underline{Z}_{0}} g_{2}+\partial_{\underline{Z}_{1}} g_{3}=0 \\
& \partial_{\underline{Z}_{1}} g_{0}+\partial_{\underline{Z}_{2}} g_{1}+\partial_{\underline{Z}_{3}} g_{2}+\partial_{\underline{Z}_{0}} g_{3}=0
\end{aligned}
$$

An important special case occurs when considering the matrix function

$$
\boldsymbol{G}_{0}=\left(\begin{array}{cccc}
g & 0 & 0 & 0 \\
0 & g & 0 & 0 \\
0 & 0 & g & 0 \\
0 & 0 & 0 & g
\end{array}\right)
$$

Indeed, $\boldsymbol{G}_{0}$ is left (resp. right) $\mathbf{Q}$-Hermitian monogenic if and only if the function $g$ is left (resp. right) $q$-Hermitian monogenic. 


\subsection{Some elements of fractal geometry}

Let $\mathbf{E}$ be an arbitrary subset of $\mathbb{R}^{4 n}$. Then for any $s \geq 0$ its Hausdorff measure $\mathcal{H}^{s}(\mathbf{E})$ may be defined by

$$
\mathcal{H}^{s}(\mathbf{E})=\liminf _{\epsilon \rightarrow 0}\left\{\sum_{k=1}^{\infty}\left(\operatorname{diam} B_{k}\right)^{s}: \mathbf{E} \subset \bigcup_{k=1}^{\infty} B_{k}, \operatorname{diam} B_{k}<\epsilon\right\}
$$

the infimum being taken over all countable $\epsilon$-coverings $\left\{B_{k}\right\}$ of $\mathbf{E}$ with open or closed balls. For $s=4 n$, the Hausdorff measure $\mathcal{H}^{4 n}$ coincides, up to a positive multiplicative constant, with the Lebesgue measure in $\mathbb{R}^{4 n}$, denoted by $\mathcal{L}$.

Now, let $\mathbf{E}$ be compact. We will use the symbol $\operatorname{dim}_{H}(\mathbf{E})$ to denote the Hausdorff dimension of $\mathbf{E}$, i.e., the infimum of all $s \geq 0$ such that $\mathcal{H}^{s}(\mathbf{E})<+\infty$. For more details concerning the Hausdorff measure and dimension we refer to [16, 17]. Frequently however, see e.g. [21], the so-called box dimension is more appropiate than the Hausdorff dimension to measure the roughness of a given set $\mathbf{E}$. The box dimension of a compact set $\mathbf{E} \subset \mathbb{R}^{4 n}$ is defined as

$$
\operatorname{dim}(\mathbf{E})=\lim _{\varepsilon \rightarrow 0} \sup \frac{\log N_{\mathbf{E}}(\varepsilon)}{-\log \varepsilon}
$$

where $\mathbf{N}_{\mathbf{E}}(\varepsilon)$ stands for the minimal number of $\varepsilon$-balls needed to cover $\mathbf{E}$. Note that the limit in (7) remains unchanged if $\mathbf{N}_{\mathbf{E}}(\varepsilon)$ is replaced by the number of $k$-cubes, with $2^{-k} \leq \varepsilon<2^{-k+1}$, intersecting $\mathbf{E}$. For completeness we recall that a cube $Q$ is called a $k$-cube if it is of the form

$$
\left[l_{1} 2^{-k},\left(l_{1}+1\right) 2^{-k}\right] \times \cdots \times\left[l_{4 n} 2^{-k},\left(l_{4 n}+1\right) 2^{-k}\right]
$$

where $k$ and $l_{1}, \ldots, l_{4 n}$ are integers. Both box and Hausdorff dimensions of a given compact set $\mathbf{E}$ can be equal, which is for instance the case for the so-called $(4 n-1)$-rectifiable sets (see [18]), but this is not true in general, where we have that $\operatorname{dim}_{H}(\mathbf{E}) \leq \operatorname{dim}(\mathbf{E})$. The following geometric notion agrees with that in [20], which was essential in their method of integrating a form over a fractal boundary.

Definition 4 The compact set $\mathbf{E}$ is said to be d-summable iff the improper integral

$$
\int_{0}^{1} N_{\mathbf{E}}(x) x^{d-1} d x
$$

converges.

Lemma 1 It holds that

(i) any d-summable set $\mathbf{E}$ has box dimension $\operatorname{dim}(\mathbf{E}) \leq d$;

(ii) if $\operatorname{dim}(\mathbf{E})<d$, then $\mathbf{E}$ is d-summable; 
(iii) if $\mathbf{E}$ is $d$-summable, then it is also $(d+\varepsilon)$-summable for every $\varepsilon>0$.

In what follows, we will take $\Omega \subset \mathbb{R}^{4 n}$ to be a Jordan domain, i.e. a bounded oriented connected open subset of $\mathbb{R}^{4 n}$, the boundary $\Gamma$ of which is a compact topological surface. For our purpose, we will assume that $\Gamma$ is $d$-summable with $d \in] 4 n-1,4 n[$.

The so-called Whitney decomposition of $\Omega$ will be needed. Consider the lattice $\mathbb{Z}^{4 n}$ in $\mathbb{R}^{4 n}$ and the collection of closed unit cubes defined by it; let $\mathcal{M}_{1}$ be the mesh consisting of those unit cubes having a non-empty intersection with $\Omega$. We then recursively define the meshes $\mathcal{M}_{k}, k=2,3, \ldots$, each time bisecting the sides of the cubes of the previous one. The cubes in $\mathcal{M}_{k}$ thus have side length $2^{-k+1}$ and diameter $|Q|=\sqrt{4 n} 2^{-k+1}$. We then define, for $k=2,3, \ldots$,

$$
\begin{array}{r}
\mathcal{W}^{1}=\left\{Q \in \mathcal{M}_{1} \mid \text { all neighbour cubes of } Q \text { belong to } \Omega\right\} \\
\mathcal{W}^{k}=\left\{Q \in \mathcal{M}_{k} \mid \text { all neighbour cubes of } Q \text { belong to } \Omega,\right. \text { and } \\
\left.\qquad Q^{*} \in \mathcal{W}^{k-1}: Q \subset Q^{*}\right\}
\end{array}
$$

for which it can be proven that

$$
\Omega=\bigcup_{k=1}^{+\infty} \mathcal{W}^{k}=\bigcup_{k=1}^{+\infty} \bigcup_{Q \in \mathcal{W}^{k}} Q \equiv \bigcup_{Q \in \mathcal{W}} Q
$$

all cubes $Q$ in the Whitney decomposition $\mathcal{W}$ of $\Omega$ having disjoint interiors.

The relation between the $d$-summability of the boundary $\Gamma$ and the Whitney decomposition of $\Omega$ is established as follows.

Lemma 2 If $\Omega$ is a Jordan domain of $\mathbb{R}^{4 n}$ and its boundary $\Gamma$ is d-summable, then the expression $\sum_{Q \in \mathcal{W}}|Q|^{d}$, called the d-sum of the Whitney decomposition $\mathcal{W}$ of $\Omega$, is finite.

For further use, we recall that, for any compact set $\mathbf{E} \subset \mathbb{R}^{4 n}$ and for any function $g \in C^{0, \alpha}(\mathbf{E})$, there exists a compactly supported function $\tilde{g} \in C^{\infty}\left(\mathbb{R}^{4 n} \backslash \mathbf{E}\right) \cap$ $C^{0, \alpha}\left(\mathbb{R}^{2 n}\right)$ for which $\left.\tilde{g}\right|_{\mathbf{E}}=g$ holds and

$$
\left|\partial_{x_{i}} \tilde{g}(\underline{X})\right| \leq c \operatorname{dist}(\underline{X}, \mathbf{E})^{\alpha-1} \quad \text { for } \underline{X} \in \mathbb{R}^{4 n} \backslash \mathbf{E}, i=1, \ldots, 4 n
$$

In fact, this extension theorem is based upon the Whitney decomposition. For our purposes, this result may be reformulated in matrix form by our next theorem.

Theorem 2 (Whitney Extension Theorem) Let $\mathbf{E} \subset \mathbb{R}^{4 n}$ be compact and $\boldsymbol{G} \in \mathcal{C}^{0, \alpha}(\mathbf{E})$. Then, there exists a compactly supported matrix function $\widetilde{\boldsymbol{G}}$ satisfying

(i) $\left.\widetilde{\boldsymbol{G}}\right|_{\mathbf{E}}=\boldsymbol{G}$;

(ii) $\widetilde{\boldsymbol{G}} \in \mathcal{C}^{\infty}\left(\mathbb{R}^{4 n} \backslash \mathbf{E}\right)$;

(iii) $\|\mathcal{D} \widetilde{\boldsymbol{G}}(\underline{X})\| \leqslant c \operatorname{dist}(\underline{X}, \mathbf{E})^{\alpha-1}$, for $\underline{X} \in \mathbb{R}^{4 n} \backslash \mathbf{E}$.

Any extension of the matrix function $\boldsymbol{G}$ satisfying the above properties will be called a Whitney type extension of $\boldsymbol{G}$. 


\subsection{The Q-Hermitian Cauchy transform on $d$-summable surfaces}

Let us introduce the following fractal version of the Cauchy type integral in the context of thematrix approach, generalizing the one introduced in [5] for the case of Lyapunov surfaces. Here, and in the sequel, $\underline{Z}=\underline{X}_{0}+i \underline{X}_{1}+j \underline{X}_{2}+k \underline{X}_{3}$ and $\underline{V}=\underline{Y}_{0}+i \underline{Y}_{1}+j \underline{Y}_{2}+k \underline{Y}_{3}$ denote the quaternionic Hermitian variables associated to $\underline{X}$ and $\underline{Y}$, repectively.

Definition 5 Let $\Omega$ be a Jordan domain in $\mathbb{R}^{4 n}$ with d-summable boundary $\Gamma$. Let $\boldsymbol{G} \in \mathcal{C}^{0, \alpha}(\Gamma)$ and $d-4 n+1<\alpha \leq 1$. Then the Hermitian Cauchy transform of $\boldsymbol{G}$ is defined by

$$
\mathcal{C}^{*}[\boldsymbol{G}](\underline{Y})=\chi_{\Omega} \widetilde{\boldsymbol{G}}(\underline{Y})+\int_{\Omega} \mathcal{E}(\underline{Z}-\underline{V})\left[\mathcal{D}^{T} \widetilde{\boldsymbol{G}}(\underline{X})\right] d \mathcal{L}(\underline{X}), \quad \underline{Y} \in \mathbb{R}^{4 n} \backslash \Gamma
$$

where

$$
\chi_{\Omega}=\operatorname{circ}\left(\begin{array}{c}
\chi_{\Omega} \\
0 \\
0 \\
0
\end{array}\right)
$$

is the matrix version of the standard characteristic function $\chi_{\Omega}$ of $\Omega$ and $\widetilde{\boldsymbol{G}}$ is a Whitney type extension of $\boldsymbol{G}$.

The integral

$$
\mathcal{T}_{\Omega} \boldsymbol{F}(\underline{Y})=-\int_{\Omega} \mathcal{E}(\underline{Z}-\underline{V}) \boldsymbol{F}(\underline{X}) d \mathcal{L}(\underline{X})
$$

is called the Teodorescu transform of $\boldsymbol{F}$.

Direct verification shows that $\mathcal{C}^{*}[\boldsymbol{G}]$ is Q-Hermitian monogenic in $\mathbb{R}^{4 n} \backslash \Gamma$, and vanishes at infinity. Definition 5 is valid, since the right hand side of (8) exists for any $\underline{Y} \in \mathbb{R}^{4 n} \backslash \Gamma$ and does not depend on the particular choice of the Whitney type extension $\widetilde{\boldsymbol{G}}$. This Hermitian Cauchy transform can be rewritten in terms of the Euclidean Cauchy type integrals given by

$$
\mathcal{C}_{r, s}^{*} g(\underline{Y})=\chi_{\Omega} \widetilde{g}(\underline{Y})+\int_{\Omega} E_{r}(\underline{X}-\underline{Y})\left[\partial_{\underline{X}_{s}} \widetilde{g}(\underline{X})\right] d \mathcal{L}(\underline{X}), \quad \underline{Y} \in \mathbb{R}^{4 n} \backslash \Gamma .
$$

Indeed,

$$
\mathcal{C}^{*}[\boldsymbol{G}]=\frac{1}{4} \operatorname{circ}\left(\begin{array}{c}
C_{0,0}^{*}+C_{1,1}^{*}+C_{2,2}^{*}+C_{3,3}^{*} \\
C_{0,0}^{*}-C_{2,2}^{*}+j\left(C_{1,3}^{*}+C_{3,1}^{*}\right) \\
C_{0,0}^{*}-C_{1,1}^{*}+C_{2,2}^{*}-C_{3,3}^{*} \\
C_{0,0}^{*}-C_{2,2}^{*}-j\left(C_{1,3}^{*}+C_{3,1}^{*}\right)
\end{array}\right)[\boldsymbol{G}]
$$


In particular, for the special case of the matrix $\boldsymbol{G}_{0}$, the action of $\mathcal{C}^{*}$ is reduced to

$$
\mathcal{C}^{*}\left[\boldsymbol{G}_{0}\right]=\frac{1}{4} \operatorname{circ}\left(\begin{array}{c}
C_{0,0}^{*} g+C_{1,1}^{*} g+C_{2,2}^{*} g+C_{3,3}^{*} g \\
C_{0,0}^{*} g-C_{2,2}^{*} g+j\left(C_{1,3}^{*} g+C_{3,1}^{*} g\right) \\
C_{0,0}^{*} g-C_{1,1}^{*} g+C_{2,2}^{*} g-C_{3,3}^{*} g \\
C_{0,0}^{*} g-C_{2,2}^{*} g-j\left(C_{1,3}^{*} g+C_{3,1}^{*} g\right)
\end{array}\right)
$$

It is clear that, in general, $\mathcal{C}^{*}\left[\boldsymbol{G}_{0}\right]$ will not be a diagonal matrix, whence its entries will not be left $q$-Hermitian monogenic functions. A natural question is whether $\mathcal{C}^{*}[\boldsymbol{G}]$ admits a continuous extension to $\bar{\Omega}=\Omega \cup \Gamma$, in which case, following the traditional structure of the Plemelj-Sokhotski formulae, a "fractal" Hermitian Hilbert transform can be introduced as follows:

$$
\mathcal{H}^{*} \boldsymbol{G}(\underline{U})=2\left(\mathcal{C}^{*} \boldsymbol{G}\right)^{+}(\underline{U})-\boldsymbol{G}(\underline{U}), \quad \underline{U} \in \Gamma,
$$

where $\left(\mathcal{C}^{*} \boldsymbol{G}\right)^{+}$denotes the trace on $\Gamma$ of the continuous extension of $\mathcal{C}^{*} \boldsymbol{G}$ to $\bar{\Omega}$.

Definition (11) would then provide an alternative for the matricial Hermitian Hilbert transform $\mathcal{H}$ introduced in [3] for domains with Lyapunov boundaries, see Theorem 2 of [4]. Now, under an additional condition on the regularity of the considered matrix function $\boldsymbol{G}$, this question indeed has an affirmative answer, as stated in the following theorem.

Theorem 3 Let $\Omega \subset \mathbb{R}^{4 n}$ with d-summable boundary $\Gamma$. Furthermore, let $d-4 n+$ $1<\alpha \leq 1$ and consider $\boldsymbol{G} \in \mathcal{C}^{0, \alpha}(\Gamma)$. If moreover

$$
\alpha>\frac{d}{4 n}
$$

then $\mathcal{C}^{*} \boldsymbol{G}(\underline{X})$ admits a continuous extension to $\bar{\Omega}$. Moreover, the Hilbert transform $\mathcal{H}^{*} \boldsymbol{G}$, defined by (11), belongs to $\mathcal{C}^{0, \mu}(\Gamma)$, for every $\mu$ such that

$$
\mu<\frac{4 n \alpha-d}{4 n-d} \text {. }
$$

Proof.

Since $\alpha>\frac{d}{4 n}$ implies that $4 n<\frac{4 n-d}{1-\alpha}$, we may choose $p$ such that $4 n<p<\frac{4 n-d}{1-\alpha}$. We will now first show that, for any such $p, \mathcal{D}^{T} \widetilde{\boldsymbol{G}} \in \boldsymbol{L}_{\boldsymbol{p}}(\Omega)$. To that end, let $\mathcal{W}=\bigcup_{k=1}^{\infty} \mathcal{W}^{k}$ be the Whitney decomposition of $\Omega$. Then we have

$$
\begin{aligned}
\int_{\Omega}\left\|\mathcal{D}^{T} \widetilde{\boldsymbol{G}}(\underline{Y})\right\|^{p} d \mathcal{L}(\underline{Y}) & =\sum_{Q \in \mathcal{W}} \int_{Q}\left\|\mathcal{D}^{T} \widetilde{\boldsymbol{G}}(\underline{Y})\right\|^{p} d \mathcal{L}(\underline{Y}) \\
& \leq c \sum_{Q \in \mathcal{W}} \int_{Q} \operatorname{dist}(\underline{Y}, \Gamma)^{-p(1-\alpha)} d \mathcal{L}(\underline{Y}),
\end{aligned}
$$

the last inequality following from Theorem 2 (iii). By construction of the Whitney decomposition of $\Omega$, we have that, for any $Q \in \mathcal{W}$,

$$
\operatorname{dist}(\underline{Y}, \Gamma) \geq \frac{|Q|}{\sqrt{4 n}}, \quad \forall \underline{Y} \in Q
$$


whence

$$
\int_{\Omega}\left\|\mathcal{D}^{T} \widetilde{\boldsymbol{G}}(\underline{Y})\right\|^{p} d \mathcal{L}(\underline{Y}) \leq c \sum_{Q \in \mathcal{W}}|Q|^{4 n-p(1-\alpha)}
$$

The finiteness of the last sum follows, on account of Lemma 2, from the $d$ summability of $\Gamma$, together with the fact that $4 n-p(1-\alpha)>d$. Hence we indeed have that $\mathcal{D}^{T} \widetilde{\boldsymbol{G}} \in \boldsymbol{L}_{\boldsymbol{p}}(\Omega)$, so that the integral term in (8), denoted by

$$
\Phi(\underline{\mathbf{Y}})=\mathcal{T}_{\Omega} \mathcal{D}^{\mathrm{T}} \widetilde{\boldsymbol{G}}(\underline{\mathbf{Y}}),
$$

represents a continuous function in $\mathbb{R}^{4 n}$, see e.g. [19]. This clearly forces $\mathcal{C}^{*} \boldsymbol{G}(\underline{X})$ to admit a continuous extension to $\bar{\Omega}$, whence $\mathcal{H}^{*} \boldsymbol{G}$ is well defined.

Moreover, $\boldsymbol{\Phi} \in \mathcal{C}^{\mathbf{0}, \frac{\mathbf{p}-\mathbf{4 n}}{\mathbf{p}}}\left(\mathbb{R}^{\mathbf{4 n}}\right)$, which implies that $\mathcal{H}^{*} \boldsymbol{G} \in \mathcal{C}^{0, \mu}(\Gamma)$ for any $\mu$ satisfying $\mu<\frac{4 n \alpha-d}{4 n-d}$.

Remark 1 Observe that, still under condition (12), we have $\left(\mathcal{C}^{*} \boldsymbol{G}\right)^{+}(\underline{U})=\boldsymbol{G}(\underline{U})-$ $\mathcal{T}_{\Omega} \mathcal{D}^{T} \widetilde{\boldsymbol{G}}(\underline{U})$ so that $\mathcal{H}^{*} \boldsymbol{G}$ may be rewritten as

$$
\mathcal{H}^{*} \boldsymbol{G}(\underline{U})=\boldsymbol{G}(\underline{U})-2 \boldsymbol{\mathcal { T }}_{\Omega} \boldsymbol{D}^{T} \widetilde{\boldsymbol{G}}(\underline{U}) .
$$

The above definition of the Quaternionic Hermitian Hilbert transform and Theorem 3 make this Hilbert transform arise naturally as part of the boundary limits of the Quaternionic Hermitian Cauchy integral, which can be written in the form of the following Plemelj-Sokhotski type formula.

Theorem 4 Let $\Omega \subset \mathbb{R}^{4 n}$ with d-summable boundary $\Gamma$. Furthermore, let $\alpha>\frac{d}{4 n}$ and consider $\boldsymbol{G} \in C^{0, \alpha}(\Gamma)$. Then the continuous limit values of $\mathcal{C}[\boldsymbol{G}]$ exist and are given by

$$
\left(\mathcal{C}^{*}\right)^{ \pm}[\boldsymbol{G}](\underline{U})=\frac{1}{2}\left(\mathcal{H}^{*}[\boldsymbol{G}](\underline{U}) \pm \boldsymbol{G}(\underline{U})\right), \underline{U} \in \Gamma .
$$

Note that Remark 1 together with the definition of the Teodorescu transform (9), allow to rewrite the Hilbert transform as the matrix operator given by

$$
\mathcal{H}^{*}[\boldsymbol{G}]=\frac{1}{4} \operatorname{circ}\left(\begin{array}{c}
H_{0,0}^{*}+H_{1,1}^{*}+H_{2,2}+H_{3,3}^{*} \\
H_{0,0}^{*}-H_{2,2}^{*}+j\left(H_{1,3}^{*}+H_{3,1}^{*}\right) \\
H_{0,0}^{*}-H_{1,1}^{*}+H_{2,2}^{*}-H_{3,3}^{*} \\
H_{0,0}^{*}-H_{2,2}^{*}-j\left(H_{1,3}^{*}+H_{3,1}^{*}\right)
\end{array}\right)[\boldsymbol{G}]
$$

where

$$
H_{r, s}^{*} g(\underline{U})=g(\underline{U})+2 \int_{\Omega} E_{r}(\underline{X}-\underline{U})\left[\partial_{\underline{X}} \widetilde{\sigma}(\underline{X})\right] d \mathcal{L}(\underline{X}), \underline{U} \in \Gamma
$$

Finally, we prove the following Q-Hermitian Cauchy integral formula. 
Theorem 5 Let $\Omega \subset \mathbb{R}^{4 n}$ with d-summable boundary $\Gamma$. Furthermore, let $d-4 n+$ $1<\alpha \leq 1$ and consider $\boldsymbol{G} \in \mathcal{C}^{0, \alpha}(\Omega \cup \Gamma)$. If $\boldsymbol{G}$ moreover is Hermitian monogenic in $\Omega$, then

$$
\mathcal{C}^{*} \boldsymbol{G}(\underline{Y})= \begin{cases}G(\underline{Y}), & \underline{Y} \in \Omega^{+} \\ 0, & \underline{Y} \in \Omega^{-}\end{cases}
$$

Proof.

Let $\widetilde{\mathbf{G}}$ be a Whitney extension of $\mathbf{G}$ and let $\mathbf{g}=\left.\mathbf{G}\right|_{\boldsymbol{\Gamma}}$. Then the function

$$
\widetilde{\widetilde{\mathbf{G}}}(\underline{Y})= \begin{cases}\widetilde{\mathbf{G}}(\underline{Y}), & \underline{Y} \in \mathbb{R}^{4 n} \backslash \Omega \\ G, & \underline{Y} \in \Omega\end{cases}
$$

is a Whithey extension of $\mathbf{g}$. Now using the definition of the Cauchy transform and the fact that it does not depend on the particular choice of the Whitney extension together with the $\mathbf{Q}$-Hermitian monogenicity of $\widetilde{\widetilde{\mathbf{G}}}=\mathbf{G}$ in $\Omega$, the integral term in (5)becomes zero and we obtain the desired formula.

\section{Boundary value problems for Q-Hermitian monogenic functions}

In this section we treat the jump problem for Q-Hermitian monogenic functions on fractal surfaces in $\mathbb{R}^{4 n}$, that is the problem of reconstructing a Q-Hermitian monogenic matrix function $\boldsymbol{\Psi}$ in $\mathbb{R}^{4 n} \backslash \Gamma$, vanishing at infinity and having a prescribed jump $\boldsymbol{G}$ across $\Gamma$, i.e.

$$
\mathbf{\Psi}^{+}(\underline{\mathbf{U}})-\boldsymbol{\Psi}^{-}(\underline{\mathbf{U}})=\boldsymbol{G}(\underline{\mathbf{U}}), \underline{\mathbf{U}} \in \boldsymbol{\Gamma},
$$

where $\Psi^{ \pm}(\underline{\mathrm{U}})$ stand for the boundary values approaching $\Gamma$ from $\Omega^{ \pm}$, respectively.

First, it should be noted that, in the general case of fractal surfaces, if this problem has a solution, then it is not necesarily unique. In particular for Lyapunov surfaces the uniqueness can be easily proved using the Painlevé and Liouville theorems in the Clifford analysis setting, see [1].

Next, if $\boldsymbol{G} \in \mathbf{C}^{0, \alpha}(\Gamma), \alpha>\frac{d}{4 n}$, a solution of this jump problem is given by

$$
\boldsymbol{\Psi}(\underline{\mathbf{Y}})=\mathcal{C}^{*} \boldsymbol{G}(\underline{\mathbf{Y}}) \text {. }
$$

Following [1], to ensure uniqueness in the general setting we need to look for the solutions of (15) in the class $\mathbf{C}^{0, \mu}$ of circulant matrix functions whose entries belong to a given space $C^{0, \mu}$, i.e., satisfy a Holder condition with exponent $\mu$ on each of the sets $\Omega^{ \pm} \cup \Gamma$.

If $\operatorname{dim}_{H}(\Gamma)-4 n+1<\mu<\frac{4 n \alpha-d}{4 n-d}$, then the solution of the jump problem in the class $\mathbf{C}^{0, \mu}$ will be unique and given by $\mathcal{C}^{*} \boldsymbol{G}(\underline{Y})$.

Now consider the special but important case of the matrix function $\boldsymbol{G}_{0}$. The above reconstruction problem (15) is then strongly connected to the similar problem for $q$-Hermitian monogenic functions. We now exploit this relation to derive the following result about the jump problem for $q$-Hermitian monogenic functions. 
Theorem 6 Let $\Omega \subset \mathbb{R}^{4 n}$ with d-summable boundary $\Gamma$. Let $\operatorname{dim}_{H}(\Gamma)-4 n+1<$ $\frac{4 n \alpha-d}{4 n-d}$ and consider $g \in C^{0, \alpha}(\Gamma)$. Then the following assertions are equivalent.

(i) The jump problem

$$
\psi^{+}(\underline{U})-\psi^{-}(\underline{U})=g(\underline{U}), \underline{U} \in \Gamma
$$

for left q-Hermitian monogenic functions has a solution in $C^{0, \mu}$ with $\operatorname{dim}_{H}(\Gamma)-$ $4 n+1<\mu<\frac{4 n \alpha-d}{4 n-d}$.

(ii) g satisfies the relations

$$
C_{0,0}^{*} g=C_{2,2}^{*} g, C_{1,3}^{*} g=-C_{3,1}^{*} g, 2 C_{0,0}^{*} g=C_{1,1}^{*} g+C_{3,3}^{*} g .
$$

(iii) $g$ satisfies the relations

$$
C_{0,0}^{*} g=C_{1,1}^{*} g=C_{2,2}^{*} g=C_{3,3}^{*} g
$$

Proof: Consider the corresponding matrix function $\boldsymbol{G}_{0}$ given by (6). By assumption, $\boldsymbol{G}_{0} \in \mathbf{C}^{0, \alpha}(\Gamma)$. Then $\mathcal{C}^{*} \boldsymbol{G}_{0}(\underline{Y})$ is a solution of (15) for $\boldsymbol{G}_{0}$ which is unique in $\mathbf{C}^{0, \mu}$ with $\operatorname{dim}_{H}(\Gamma)-4 n+1<\mu<\frac{4 n \alpha-d}{4 n-d}$.

$(i) \rightarrow(i i)$

Let $\psi$ be a solution of (16), then the circulant matrix

$$
\operatorname{circ}\left(\begin{array}{l}
\psi \\
0 \\
0 \\
0
\end{array}\right)
$$

is another solution of the jump problem (15) for $\boldsymbol{G}_{0}$ in $\mathbf{C}^{0, \mu}$ and hence, by uniqueness,

$$
\operatorname{circ}\left(\begin{array}{c}
\psi \\
0 \\
0 \\
0
\end{array}\right)=\frac{1}{4} \operatorname{circ}\left(\begin{array}{c}
C_{0,0}^{*} g+C_{1,1}^{*} g+C_{2,2}^{*} g+C_{3,3}^{*} g \\
C_{0,0}^{*} g-C_{2,2}^{*} g+j\left(C_{1,3}^{*} g+C_{3,1}^{*} g\right) \\
C_{0,0}^{*} g-C_{1,1}^{*} g+C_{2,2}^{*} g-C_{3,3}^{*} g \\
C_{0,0}^{*} g-C_{2,2}^{*} g-j\left(C_{1,3}^{*} g+C_{3,1}^{*} g\right)
\end{array}\right)
$$

which implies $(i i)$.

$($ ii $) \rightarrow($ iii $)$

From the third relation in (17) we have

$$
2 \partial_{\underline{Y}_{1}} C_{0,0}^{*} g=\partial_{\underline{Y}_{1}} C_{3,3}^{*} g+\partial_{\underline{Y}_{1}} C_{1,1}^{*} g=\partial_{\underline{Y}_{1}} C_{3,3}^{*}
$$

and hence

$$
2 \partial_{\underline{Y}_{1}} C_{0,0}^{*} g=\partial_{\underline{Y}_{1}} C_{3,3}^{*} g=-\partial_{\underline{Y}_{3}} C_{1,3}^{*} g=\partial_{\underline{Y}_{3}} C_{3,1}^{*} g=0,
$$

which is a consequence of the second relation in (17) and the obvious $\partial_{\underline{Y}_{3}}$-monogenicity of $C_{3,1}^{*} g$. 
This fact means that the function $\left(C_{0,0}^{*} g-C_{3,3}^{*} g\right) \in C^{0, \mu}$, being $\partial_{\underline{Y}_{1}}$-monogenic in $\mathbb{R}^{4 n} \backslash \Gamma$, has a null jump through $\Gamma$. Then, by uniqueness, $C_{0,0}^{*} g=C_{1,1}^{*} g$.

The same argument gives $C_{0,0}^{*} g \equiv C_{3,3}^{*} g$. $($ iii $) \rightarrow($ i $)$

Note that, under the given assumptions, $C_{0,0}^{*} g$, being $q$-Hermitian monogenic, is the solution of (16).

Similar arguments apply to right $q$-Hermitian monogenic functions.

Theorem 7 Let $\Omega \subset \mathbb{R}^{4 n}$ with d-summable boundary $\Gamma$. Let $\operatorname{dim}_{H}(\Gamma)-4 n+1<$ $\frac{4 n \alpha-d}{4 n-d}$ and consider $g \in C^{0, \alpha}(\Gamma)$. Then the following assertions are equivalent.

(i) The jump problem

$$
\psi^{+}(\underline{U})-\psi^{-}(\underline{U})=g(\underline{U}), \underline{U} \in \Gamma
$$

for right q-Hermitian monogenic functions has a solution in $C^{0, \mu}$ with $\operatorname{dim}_{H}(\Gamma)-$ $4 n+1<\mu<\frac{4 n \alpha-d}{4 n-d}$.

(ii) g satisfies the relations

$$
g C_{0,0}^{*}=g C_{2,2}^{*}, g C_{1,3}^{*}=-g C_{3,1}^{*}, 2 g C_{0,0}^{*}=g C_{1,1}^{*}+g C_{3,3}^{*}
$$

(iii) $g$ satisfies the relations

$$
g C_{0,0}^{*}=g C_{1,1}^{*}=g C_{2,2}^{*}=g C_{3,3}^{*}
$$

\subsection{Dirichlet problem for Q-Hermitian monogenic func- tions}

Theorem 8 Let $\Omega \in \mathbb{R}^{4 n}$ with d-summable boundary $\Gamma$. Consider $\boldsymbol{G} \in \mathbf{C}^{0, \alpha}(\Gamma)$ with $\alpha>\frac{d}{4 n}$. Then the following are equivalent.

(i) The Dirichlet problem

$$
\begin{array}{r}
\mathcal{D}^{T} \mathbf{F}=\boldsymbol{O}\left(\text { resp. } \mathbf{F} \mathcal{D}^{T}=\boldsymbol{O}\right), \\
\mathbf{F}=\boldsymbol{G}, \text { in } \Omega
\end{array}
$$

has a solution.

(ii) $\mathcal{H}^{*}[\boldsymbol{G}]=\boldsymbol{G}\left(\right.$ resp. $\left.[\boldsymbol{G}] \mathcal{H}^{*}=\boldsymbol{G}\right)$

\section{Proof:}

$(i) \rightarrow(i i)$

Let $\mathbf{F}$ be a solution of the Dirichlet problem (19). Then, by the $\mathbf{Q}$-Hermitian Cauchy formula (14), we have

$$
\mathcal{C}^{*}[\mathbf{F}](\underline{Y})=\mathbf{F}(\underline{Y}), \underline{Y} \in \Omega .
$$


Now (ii) follows by taking limits for $\underline{Y} \rightarrow \underline{U} \in \Gamma$ and on account of the formulae for the boundary values of the Cauchy transform in terms of the fractal Hilbert transform.

$($ ii $) \rightarrow(i)$ Note that, under the condition $(i i), \mathbf{F}=\mathcal{C}^{*}[\boldsymbol{G}]$ is a solution of (19).

Theorem 9 Let $\Omega \subset \mathbb{R}^{4 n}$ with d-summable boundary $\Gamma$. Let $\operatorname{dim}_{H}(\Gamma)-4 n+1<$ $\frac{4 n \alpha-d}{4 n-d}$ and consider $g \in C^{0, \alpha}(\Gamma)$. The following are equivalent.

(i) The Dirichlet problem

$$
\begin{aligned}
\partial_{\underline{Z}_{0}} f=\partial_{\underline{Z}_{1}} f=\partial_{\underline{Z}_{2}} f=\partial_{\underline{Z}_{3}} f & =0, \text { in } \Omega \\
f & =g, \text { on } \Gamma
\end{aligned}
$$

has a solution.

(ii) $g$ satisfies the relations

$$
H_{0,0}^{*} g=H_{2,2}^{*} g=g, H_{1,3}^{*} g=-H_{3,1}^{*} g, H_{1,1}^{*} g+H_{3,3}^{*} g=2 g
$$

(iii) $g$ satisfies the relations

$$
H_{0,0}^{*} g=H_{1,1}^{*} g=H_{2,2}^{*} g=H_{3,3}^{*} g=g
$$

\section{Proof:}

$(1) \rightarrow(2)$

From (1) we see that the matrix function

$$
\mathbf{F}_{0}=\operatorname{circ}\left(\begin{array}{c}
f \\
0 \\
0 \\
0
\end{array}\right)
$$

is a solution of the Dirichlet problem

$$
\begin{array}{r}
\mathcal{D}^{T} \mathbf{F}=\boldsymbol{O}, \text { in } \Omega \\
\mathbf{F}=\boldsymbol{G}_{0}, \text { on } \Gamma
\end{array}
$$

and hence, by Theorem 8 , we have $\mathcal{H}^{*}\left[\boldsymbol{G}_{0}\right]=\boldsymbol{G}_{0}$. The desired conclusion (ii) follows directly by comparing the entries in the above matrix equality.

$($ ii $) \rightarrow($ iii $)$

We see from the condition $H_{0,0}^{*} g=H_{2,2}^{*} g=g$ that $\left(C_{0,0}^{*}\right)^{ \pm} g=\left(C_{2,2}^{*}\right)^{ \pm} g$. Therefore, as $C_{0,0}^{*} g-C_{2,2}^{*} g$ is harmonic in $\Omega^{ \pm}$and $\left(C_{0,0}^{*}\right)^{ \pm} g-\left.\left(C_{2,2}^{*}\right)^{ \pm} g\right|_{\Gamma}=0$, we have $C_{0,0}^{*} g=C_{2,2}^{*} g$ in $\mathbb{R}^{4 n} \backslash \Gamma$.

Using the remaining conditions in (ii) and proceeding in a similar way as above, we obtain that $g$ satisfies the relations (17) and hence, by Theorem 6 , we have

$$
C_{0,0}^{*} g=C_{1,1}^{*} g=C_{2,2}^{*} g=C_{3,3}^{*} g .
$$


Consequently,

$$
H_{0,0}^{*} g=H_{1,1}^{*} g=H_{2,2}^{*} g=H_{3,3}^{*} g=g,
$$

as desired.

$($ iii $) \rightarrow(i)$

Conditions

$$
H_{0,0}^{*} g=H_{1,1}^{*} g=H_{2,2}^{*} g=H_{3,3}^{*} g=g,
$$

imply the solvability of the Dirichlet problems

$$
\begin{aligned}
\partial_{\underline{X}_{r}} f & =0, \text { in } \Omega \\
f & =g, \text { on } \Gamma
\end{aligned}
$$

where $r=0, \ldots, 3$.

Let $f_{0}, f_{1}, f_{2}, f_{3}$ be the respective solutions of (21). Then, naturally, all these functions will be simultaneously solutions of the classical Dirichlet problem

$$
\begin{gathered}
\Delta_{4 n} f=0, \text { in } \Omega \\
f=g, \text { on } \Gamma .
\end{gathered}
$$

It thus suffices to see that $f_{0}=f_{1}=f_{2}=f_{3}$ gives a solution of (20).

For right $q$-Hermitian monogenic functions the following analogue is obtained.

Theorem 10 Let $\Omega \subset \mathbb{R}^{4 n}$ with d-summable boundarz $\Gamma$. Let $\operatorname{dim}_{H}(\Gamma)-4 n+1<$ $\frac{4 n \alpha-d}{4 n-d}$ and consider $g \in C^{0, \alpha}(\Gamma)$. The following are equivalent.

(i) The Dirichlet problem

$$
\begin{array}{r}
f \partial_{\underline{Z}_{0}}=f \partial_{\underline{Z}_{1}}=f \partial_{\underline{Z}_{2}}=f \partial_{\underline{Z}_{3}}=0, \\
f=g, \text { in } \Omega \\
\text { on } \Gamma
\end{array}
$$

has a solution.

(ii) $g$ satisfies the relations

$$
g H_{0,0}^{*}=g H_{2,2}^{*}=g, g H_{1,3}^{*}=-g H_{3,1}^{*}, g H_{1,1}^{*}+g H_{3,3}^{*}=2 g
$$

(iii) $g$ satisfies the relations

$$
g H_{0,0}^{*}=g H_{1,1}^{*}=g H_{2,2}^{*}=g H_{3,3}^{*} g=g
$$

\subsection{Criteria for two-sided Q-Hermitian monogenicity}

We now establish a connection between two-sided Q-Hermitian monogenicity of a matrix function $\boldsymbol{G}$ and the matrix Hilbert transforms $\mathcal{H}^{*}\left[\left.\boldsymbol{G}\right|_{\Gamma}\right]$ and $\left[\left.\boldsymbol{G}\right|_{\Gamma}\right] \mathcal{H}^{*}$ of its trace on the boundary $\Gamma$. 
Theorem 11 Let $\Omega \subset \mathbb{R}^{4 n}$ with d-summable boundary $\Gamma$. Suppose $\boldsymbol{G} \in \mathbf{C}^{0, \alpha}(\Omega \cup$ $\Gamma), \alpha>\frac{d}{4 n}$, such that $\mathcal{D}^{T} \boldsymbol{G}=\boldsymbol{O}$ in $\Omega$. Then the following are equivalent.

(i) $\boldsymbol{G}$ is two-sided $\mathbf{Q}$-Hermitian monogenic in $\Omega$

(ii) $\mathcal{H}^{*}\left[\left.\boldsymbol{G}\right|_{\Gamma}\right]=\left[\left.\boldsymbol{G}\right|_{\Gamma}\right] \mathcal{H}^{*}$

Proof: Suppose that, as assumed, $\boldsymbol{G}$ is not only left $\mathbf{Q}$-Hermitian monogenic, but also right $\mathbf{Q}$-Hermitian monogenic in $\Omega$. Then by Theorem 8 it holds that

$$
\mathcal{H}^{*}\left[\left.\boldsymbol{G}\right|_{\Gamma}\right]=\left.\boldsymbol{G}\right|_{\Gamma}=\left[\left.\boldsymbol{G}\right|_{\Gamma}\right] \mathcal{H}^{*}
$$

Conversely, suppose that $\mathcal{H}^{*}\left[\left.\boldsymbol{G}\right|_{\Gamma}\right]=\left[\left.\boldsymbol{G}\right|_{\Gamma}\right] \mathcal{H}^{*}$. By Theorem 4 an its right-handed version, we conclude that the corresponding left and right Q-Hermitian Cauchy transforms of $\boldsymbol{G}, \mathcal{C}^{*}[\boldsymbol{G}]$ and $[\boldsymbol{G}] \mathcal{C}^{*}$, have the same boundary values on $\Gamma$. This fact, together with their harmonicity, imply

$$
\mathcal{C}^{*}[\boldsymbol{G}]=[\boldsymbol{G}] \mathcal{C}^{*}
$$

On the other hand, from the assumed left Q-Hermitian monogenicity of $\boldsymbol{G}$ we have $\boldsymbol{G}=\mathcal{C}^{*}[\boldsymbol{G}]$ and hence

$$
\boldsymbol{G}=\mathcal{C}^{*}[\boldsymbol{G}]=[\boldsymbol{G}] \mathcal{C}^{*},
$$

which clearly forces $\boldsymbol{G}$ to be two-sided $\mathbf{Q}$-Hermitian monogenic.

The following result illustrates the usefulness of the above theorem when considering $q$-Hermitian monogenic functions.

Theorem 12 Let $\Omega \subset \mathbb{R}^{4 n}$ with d-summable boundary $\Gamma$. Suppose $\alpha>\frac{d}{4 n}, g \in$ $C^{0, \alpha}(\Omega \cup \Gamma)$ and $g$ q-Hermitian monogenic in $\Omega$. Then the following are equivalent.

(i) $g$ is two-sided q-Hermitian monogenic in $\Omega$

(ii) g satisfies the relations

$$
\begin{gathered}
H_{0,0}^{*} g=g H_{0,0}^{*}, H_{2,2}^{*} g=g H_{2,2}^{*} \\
H_{1,3}^{*} g+H_{3,1}^{*} g=g H_{1,3}^{*}+g H_{3,1}^{*} \\
H_{1,1}^{*} g+H_{3,3}^{*} g=g H_{1,1}^{*}+g H_{3,3}^{*}
\end{gathered}
$$

(iii) $g$ satisfies the relations

$$
H_{0,0}^{*} g=g H_{0,0}^{*}, H_{1,1}^{*} g=g H_{1,1}^{*}, H_{2,2}^{*} g=g H_{2,2}^{*}, H_{3,3}^{*} g=g H_{3,3}^{*}
$$

\section{Proof:}

First, let us prove $(i) \leftrightarrow(i i)$. From $(i)$ we see that the corresponding matrix function

$$
\boldsymbol{G}_{0}=\operatorname{circ}\left(\begin{array}{l}
g \\
0 \\
0 \\
0
\end{array}\right)
$$


is two-sided $\mathbf{Q}$-Hermitian monogenic in $\Omega$. Now (ii) follows from Theorem 11(ii) applied to $\boldsymbol{G}_{0}$.

On the other hand, conditions (ii) can be rewritten in the matricial form $\mathcal{H}^{*}\left[\left.\boldsymbol{G}_{0}\right|_{\Gamma}\right]=\left[\left.\boldsymbol{G}_{0}\right|_{\Gamma}\right] \mathcal{H}^{*}$. The desired conclusion $(i)$ follows after noting that the two-sided Q-Hermitian monogenicity of $\boldsymbol{G}_{0}$, implied by Theorem 11, is equivalent to the $q$-Hermitian monogenicity of $g$.

Next, let us prove that $(i) \leftrightarrow($ iii $)$

It follows from $(i)$ that $g$ is two-sided monogenic w.r.t $\partial_{\underline{X}_{r}}(r=0, \ldots, 3)$. At this stage we appeal to Theorem 3.3 in [6] to deduce that $H_{r, r}^{*} g=g H_{r, r}^{*}$ $(r=0, \ldots, 3)$. Conversely, suppose that (iii) holds. Each condition $H_{r, r}^{*} g=g H_{r, r}^{*}$ $(r=0, \ldots, 3)$ implies the two sided monogenicity of $g$ in $\Omega$ w.r.t $\partial_{\underline{X}_{r}}(r=0, \ldots, 3)$, see again Theorem 3.3 in [6]. It folllows that $g$ is two-sided $q$-Hermitian monogenic in $\Omega$.

\section{Acknowledgments}

This article was written during a scientific stay of R. Abreu-Blaya, J. Bory-Reyes and Tania Moreno-García at the Clifford Research Group of the Department of Mathematical Analysis of Ghent University; the financial support and kind hospitality are gratefully acknowledged.

\section{References}

[1] R. Abreu Blaya; J. Bory Reyes; D. Peña Peña. Jump problem and removable singularities for monogenic functions. J. Geom. Anal. 17 (2007), no. 1, 1-13.

[2] R. Abreu Blaya; J. Bory Reyes; Brackx, F.; De Schepper and F. Sommen, Cauchy Integral Formulae in Hermitian Quaternionic Clifford Analysis. Compl. Anal. Oper. Theory, (2011), doi: 10.1007/s11785-011-0168-8.

[3] R. Abreu Blaya; J. Bory Reyes; Brackx, F.; De Schepper and F. Sommen, Matrix Cauchy and Hilbert transforms in Hermitian quaternionic Clifford analysis. Complex Var. Elliptic Equ., (2011), doi: 10.1080/17476933.2011.626034.

[4] R. Abreu Blaya; J. Bory Reyes; Brackx, F.; De Schepper and F. Sommen, A Hilbert transform for matrix functions on fractal domains. Compl. Anal. Oper. Theory vol. 6, no. 2, 359-372, 2012.

[5] R. Abreu Blaya; J. Bory Reyes; F. Brackx; H. De Schepper and F. Sommen, Boundary value problems for the quaternionic Hermitian system in $\mathbb{R}^{4 n}$. To appear in Boundary Value Problems. 
[6] R. Abreu Blaya; J. Bory Reyes; T. Moreno García. Teodorescu transform decomposition of multivector fields on fractal hypersurfaces. Wavelets, multiscale systems and hypercomplex analysis, 1-16, Oper. Theory Adv. Appl., 167, Birkhuser, Basel, 2006.

[7] F. Brackx; R. Delanghe; F. Sommen. Clifford Analysis. Research Notes in Mathematics, 76. Pitman, Boston, MA, 1982.

[8] F. Brackx; J. Bureš; H. De Schepper; D. Eelbode; F. Sommen; V. Souček. $F$ undaments of Hermitian Clifford analysis. I. Complex structure. Complex Anal. Oper. Theory 1 (2007), no. 3, 341-365.

[9] F. Brackx; J. Bureš; H. De Schepper; D. Eelbode; F. Sommen; V. Souček. $F$ undaments of Hermitian Clifford analysis. II. Splitting of $h$-monogenic equations. Complex Var. Elliptic Equ. 52 (2007), no. 10-11, 1063-1079.

[10] F. Brackx; H. De Schepper; N. De Schepper; F. Sommen. Hermitian CliffordHermite wavelets: an alternative approach. Bulletin of the Belgian mathematical Society-Simon Stevin, Volume: 15 Issue: 1 Pages: 87-107, 2008

[11] F. Brackx; H. De Schepper; F. Sommen. The Hermitian Clifford analysis toolbox. Adv. Appl. Clifford Algebras, 18 (2008), no. 3-4, 451-487.

[12] F. Brackx, B. De Knock, H. De Schepper. A matrix Hilbert transform in Hermitian Clifford analysis. J. Math. Anal. Appl. 344 (2008) 1068-1078

[13] A. Damiano, E. Eelbode, I. Sabadini. Quaternionic Hermitian spinor systems and compatibility conditions. Adv. Geom. 11 (2011), 169-189

[14] A. Damiano, E. Eelbode, I. Sabadini. Algebraic analysis of Hermitian monogenic functions, C. R. Acad. Sci. Paris, Ser. I 346 (2008) 139-142

[15] D. Eelbode. A Clifford algebraic framework for sp(m)-invariant differential operators. Adv. Appl. Clifford Algebr. 17 (2007), 635649.

[16] K.J. Falconer, The geometry of fractal sets, Cambridge Tracts in Mathematics 85, Cambridge University Press, Cambridge, 1986.

[17] J. Feder, Fractals (With a foreword by Benoit B. Mandelbrot), Physics of Solids and Liquids, Plenum Press, New York, 1988.

[18] H. Federer, Geometric measure theory, Die Grundlehren der mathematischen Wissenschaften 153, Springer Verlag, New York Inc., New York, 1969.

[19] K. Gürlebeck, K. Habetha, W. Sprössig, Holomorphic functions in the plane and n-dimensional space, translated from the 2006 German original, Birkhäuser Verlag, Basel, 2008. 
[20] J. Harrison, A. Norton, The Gauss-Green theorem for fractal boundaries, Duke Mathematical Journal 67(3) (1992), 575-588.

[21] M.L. Lapidus, H. Maier, Hypothse de Riemann, cordes fractales vibrantes et conjecture de Weyl-Berry modifie (French), C.R. Acad. Sci. Paris Série I Math. 313(1) (1991), 19-24.

$[22]$ D. Peña-Peña, I. Sabadini, F. Sommen. Quaternionic Clifford analysis: the Hermitian setting. Complex Anal. Oper. Theory 1 (2007), 97-113.

[23] I. Sabadini; F. Sommen. Hermitian Clifford analysis and resolutions. Clifford analysis in applications. Math. Methods Appl. Sci. 25 (2002), no. 16-18, $1395-1413$. 